\title{
Finiteness of spatial central configurations in the five-body problem
}

\author{
Marshall Hampton • Anders Jensen
}

Received: 12 August 2010 / Revised: 22 November 2010 / Accepted: 8 December 2010 /

Published online: 8 January 2011

(C) The Author(s) 2011. This article is published with open access at Springerlink.com

\begin{abstract}
We strengthen a generic finiteness result due to Moeckel by showing that the number of spatial central configurations of the Newtonian five-body problem with positive masses is finite, apart from some explicitly given special cases of mass values.
\end{abstract}

Keywords Central configurations · n-Body problem · Tropical geometry · Polyhedral fan · Albouy-Chenciner equations

\section{Introduction}

In this paper we present a computer-assisted proof of the finiteness of the spatial central configurations of the Newtonian five-body problem with positive masses, with the exception of some explicit special cases of mass values.

By the Newtonian spatial $n$-body problem we mean the dynamical system given by

$$
m_{j} \ddot{x}_{j}=\sum_{i \neq j} \frac{m_{i} m_{j}\left(x_{i}-x_{j}\right)}{r_{i j}^{3}} \quad 1 \leq j \leq n
$$

where $x_{i} \in \mathbb{R}^{3}$ is the position of particle $i, r_{i j}$ is the distance between $x_{i}$ and $x_{j}$, and $m_{i}$ is the mass of particle $i$ (Newton 1687).

\footnotetext{
M. Hampton $(\varangle)$

Department of Mathematics and Statistics, University of Minnesota, 1117 University Drive, Duluth, MN 55812, USA

e-mail:mhampton@d.umn.edu

A. Jensen

Mathematisches Institut, Georg-August-Universität Göttingen, Bunsenstr. 3-5,

37073 Göttingen, Germany

e-mail: jensen@uni-math.gwdg.de
} 
A central configuration of the $n$-body satisfies the equations

$$
\lambda\left(x_{j}-c\right)=\sum_{i \neq j} \frac{m_{i}\left(x_{i}-x_{j}\right)}{r_{i j}^{3}} \quad 1 \leq j \leq n
$$

where $\lambda<0$ and $c$ is the center of mass. Such a configuration, if started from rest, will collapse to the center of mass. In the planar case these configurations also give rise to relative equilibria - orbits in which each particle moves on a circle at a common angular speed.

For the Newtonian three-body and four-body problems it is known that central configurations in dimensions one, two, and three are finite for positive masses (Euler 1767; Lagrange 1772; Moulton 1910; Hampton and Moeckel 2006). There is also a generic finiteness result for 'Dziobek configurations' which applies to the case we are studying (Moeckel 2001) since the dimension of our configurations is $n-2$.

The five-body problem is of particularly interest in this context because of the known continuum of planar central configurations for the five-body problem if one negative mass is permitted (Roberts 1999). Recently Alain Albouy and Vadim Kaloshin have proved that the planar five-body central configurations are finite apart from some explicitly given special cases (Albouy and Kaloshin 2010).

We have proven (with computer assistance on exact computations) the following result:

Theorem 1 There are finitely many central configurations of the Newtonian spatial five-body problem with the possible exception of some mass parameter values on which an explicitly given set of polynomials vanish (see Table 1 in Sect. 4).

The proof of this theorem is given by the remainder of our paper. In Sect. 2 we briefly review the necessary tropical geometry. Following that we describe the equations we use in Sect. 3. The exceptional cases are given in Table 1 of Sect. 4 along with a description of the tropical prevariety of our equations. The next two Sects. 5 and 6 give details of the two most complicated cases that arose from the tropical prevariety.

\section{Tropical geometry}

Our proof strategy will be the same as that of Hampton and Moeckel (2006), but where they use the theory of Bernstein (1975), Khovanskii (1977), and Kushnirenko (1976) we will use the language of tropical geometry. Our equations for central configurations (described in Sect. 3) define an algebraic variety $V(I)$ in the algebraic torus $\left(\mathbb{C}^{*}\right)^{10}=(\mathbb{C} \backslash\{0\})^{10}$. Instead of proving that the algebraic variety has dimension 0 we will attempt to prove that its tropical variety is a polyhedral fan of dimension 0 . Both varieties depend on the choice of masses. We will consider these masses as being unknown positive real numbers. In this section we give the necessary definitions in tropical geometry and refer to Sturmfels (2002, Chapter 9) for a general introduction. The primary purpose of this section is to introduce terminology, but we will also see that Hampton and Moeckel (2006, Proposition 2) falls out of the general theory.

Definition 1 Let $I \subseteq \mathbb{C}\left[x_{1}, \ldots, x_{n}\right]$ be an ideal and $\omega \in \mathbb{R}^{n}$ a vector. For a monomial $x^{v}:=x_{1}^{v_{1}} \cdots x_{n}^{v_{n}}$ with $v \in \mathbb{N}^{n}$ we define its $\omega$-degree as $\omega \cdot v$. The initial form $\operatorname{in}_{\omega}(f)$ of a polynomial $f \in \mathbb{C}\left[x_{1}, \ldots, x_{n}\right]$ is the sum of all terms of $f$ with maximal $\omega$-degree. The initial ideal of $I$ with respect to $\omega$ is defined as $\operatorname{in}_{\omega}(I):=\left\langle\operatorname{in}_{\omega}(f): f \in I\right\rangle$.

We note that the initial ideal is not always a monomial ideal. 
Definition 2 The tropical variety of an ideal $I \subseteq \mathbb{C}\left[x_{1}, \ldots, x_{n}\right]$ is the set

$$
T(I):=\left\{\omega \in \mathbb{R}^{n}: \operatorname{in}_{\omega}(I) \text { does not contain a monomial }\right\} .
$$

For an ideal $J \subseteq \mathbb{C}\left[x_{1}, \ldots, x_{n}\right]$ and a polynomial $f \in \mathbb{C}\left[x_{1}, \ldots, x_{n}\right]$ we define the saturation $\left(J: f^{\infty}\right):=\left\{g \in \mathbb{C}\left[x_{1}, \ldots, x_{n}\right]: \exists m: g f^{m} \in J\right\}$. The saturation $\left(\operatorname{in}_{\omega}(I): x_{1} \cdots x_{n}^{\infty}\right)$ is $\langle 1\rangle$ if and only if $\operatorname{in}_{\omega}(I)$ contains a monomial which is the case if and only if $\omega \notin T(I)$.

A polyhedral fan $F$ is a finite collection of polyhedral cones such that if $C \in F$ then every face $C$ is in $F$ and if $C, C^{\prime} \in F$ then $C \cap C^{\prime}$ is a face of $C$. The support of a fan is the union of its cones. If all inclusion-maximal cones of $F$ are of the same dimension $d$, then $F$ is called pure of dimension $d$. A subfan of $F$ is a subset of $F$ which is itself a fan. For further background on the geometry of polyhedral fans we refer the reader to Zeigler (1995).

The tropical variety $T(I)$ can be thought of as a polyhedral fan. Namely, two vectors in $\mathbb{R}^{n}$ are considered equivalent if they give the same initial ideal $\operatorname{in}_{\omega}(I)$. If $I$ is homogeneous, then the equivalence classes are convex and their closures form the Gröbner fan of $I$. The subfan consisting of cones with monomial free initial ideals has $T(I)$ as support.

The following theorem was first stated by Bieri and Groves (1984) in terms of valuations. A proof in the language of initial ideals was given in Sturmfels (2002), see also Jensen (2007, Chapter 8). It will be essential for our arguments.

Theorem 2 (Bieri Groves) Let $I \subseteq \mathbb{C}\left[x_{1}, \ldots, x_{n}\right]$ be a prime ideal and let $d$ be the dimension of the variety $V(I) \subseteq\left(\mathbb{C}^{*}\right)^{n}$ defined by $I$. The tropical variety $T(I)$ is the support of a pure d-dimensional polyhedral fan.

If $I$ is not a prime ideal, then by the Lasker-Noether theorem we may find a primary decomposition of $I$ i.e. write $I$ as $\bigcap_{i} Q_{i}$ with the radicals $\sqrt{Q_{i}}$ being prime ideals. It is not difficult to prove that $T(I)=\bigcup T\left(\sqrt{Q_{i}}\right)$, see for example Jensen (2007). Therefore, applying Theorem 2 to each $\sqrt{Q_{i}}$, we conclude that the dimension of $V(I)$ is the largest dimension of a cone in $T(I)$.

For our purpose it will suffice to compute $T(I)$ and see that it is zero-dimensional to conclude that $V(I)$ is zero-dimensional. A first approximation to $T(I)$ comes from the following description, see Sturmfels (2002).

Proposition 1 Let $I \subseteq \mathbb{C}\left[x_{1}, \ldots, x_{n}\right]$. The following identity holds

$$
T(I)=\bigcap_{f \in I} T(\langle f\rangle) .
$$

Given a set of generators $f_{1}, \ldots, f_{m}$ of $I$ we may compute the tropical prevariety $\bigcap_{i} T\left(\left\langle f_{i}\right\rangle\right)$ defined by $f_{1}, \ldots, f_{m}$. However, the prevariety does not equal $T(I)$ in general. The software Gfan (Jensen 2009) will compute the prevariety as a polyhedral fan. For each cone in this fan of positive dimension we wish to check if the cone is contained in $T(I)$. It is possible that some relative interior points are in $T(I)$ and others are not. To check if a single ray $\omega$ is contained in $T(I)$ we can in theory compute in $_{\omega}(I)$. However, computing initial ideals is usually done by computing a Gröbner basis (Sturmfels 1996, Corollary 1.9). This computation is not feasible in our setting. Indeed, if we could compute a Gröbner basis for $I$ we would also know the dimension of $I$.

Instead we will approximate $\operatorname{in}_{\omega}(I)$ by choosing a big generating set for $I$ and taking initial forms with respect to $\omega$. If the ideal generated by these initial forms contains a monomial, then so does $\operatorname{in}_{\omega}(I)$.

It is a feature of $T(I)$ that it ignores how $V(I)$ looks in the coordinate hyperplanes. That is, in algebraic terms, $T(I)=T\left(\left(I: x_{1} \cdots x_{n}^{\infty}\right)\right)$. Therefore, it makes sense to let tropical 
varieties be defined by ideals in the Laurent polynomial ring $\mathbb{C}\left[x_{1}^{ \pm 1}, \ldots, x_{n}^{ \pm 1}\right]$ instead. One advantage of this is that we can make multiplicative changes of coordinates in the following sense: For $A \in G L_{n}(\mathbb{Z})$ we let $f \mapsto f\left(x^{A_{1}}, \ldots, x^{A_{n} \cdot}\right)$. Transforming an ideal has the effect of making a linear transformation of its tropical variety.

We need two more properties of tropical varieties. First, tropical varieties are balanced which implies that a tropical variety in 1-dimensional space $\mathbb{R}$ is either all of $\mathbb{R}$, empty or the origin (corresponding to a principal ideal having, respectively, 0,1 or more monomials in its generator). Second, a rational projection of a tropical variety to a linear subspace is again a tropical variety. This follows from Hept and Theobald (2009, Theorem 3.1) and the possibility to do multiplicative changes of coordinates in the Laurent polynomial ring. Putting this together we get that to show that a tropical variety $T(I)$ equals the origin, it suffices to show that $T(I) \cap\left\{\omega \in \mathbb{R}^{n}: \sum_{i} \omega_{i} \geq 0\right\}=\{0\}$. The fundamental theorem of tropical geometry (Speyer and Sturmfels 2004, Theorem 2.1) connects tropical varieties to Puiseux series and shows that the last statement is indeed equivalent to Hampton and Moeckel (2006, Proposition 2).

\section{Equations for the spatial case}

We will use two versions of the Albouy-Chenciner equations for central configurations (originally described in Albouy and Chenciner 1998). The first version is identical to that discussed in Hampton and Moeckel (2006), namely the polynomial versions (with denominators cleared) of

$$
f_{i j}=\sum_{k=1}^{n} m_{k}\left[S_{i k}\left(r_{j k}^{2}-r_{i k}^{2}-r_{i j}^{2}\right)+S_{j k}\left(r_{i k}^{2}-r_{j k}^{2}-r_{i j}^{2}\right)\right]=0
$$

where $S_{i j}=r_{i j}^{-3}+\Lambda$. We choose to set $\Lambda=-1$, which can be done without loss of generality because of the homogeneity of the equations above. We will refer to these as the symmetric Albouy-Chenciner equations. We will denote the complete set of the $f_{i j}$ as $\mathcal{F}$.

Gareth Roberts observed (Roberts 2009) that a more restrictive set of equations can be obtained from the Albouy-Chenciner linear operator equations, namely

$$
g_{i j}=\sum_{k=1}^{n} m_{k} S_{i k}\left(r_{j k}^{2}-r_{i k}^{2}-r_{i j}^{2}\right)=0 .
$$

Since $g_{i j} \neq g_{j i}$, these give 20 equations in the five-body problem. Since $f_{i j}=g_{i j}+g_{j i}$ the $f_{i j}$ equations are redundant but they were included in the tropical intersection calculation anyway in order to generate a more refined set of cones. These equations will collectively be denoted as $\mathcal{G}$.

Fifteen more 'Dziobek' equations were added (Dziobek 1900). Only five of these are independent, but all were included to preserve symmetry:

$$
h_{i j k l}=\left(r_{i j}^{-3}-1\right)\left(r_{k l}^{-3}-1\right)-\left(r_{i k}^{-3}-1\right)\left(r_{j l}^{-3}-1\right)=0
$$

where $i, j, k$, and $l$ are distinct indices. The set of Dziobek equations will be denoted $\mathcal{H}$. 
The distances between five points in $\mathbb{R}^{3}$ satisfy a single constraint, that the determinant of the Cayley-Menger matrix is zero:

$$
e_{C M}=\operatorname{det}\left(\begin{array}{cccccc}
0 & 1 & 1 & 1 & 1 & 1 \\
1 & 0 & r_{12}^{2} & r_{13}^{2} & r_{14}^{2} & r_{15}^{2} \\
1 & r_{12}^{2} & 0 & r_{23}^{2} & r_{24}^{2} & r_{25}^{2} \\
1 & r_{13}^{2} & r_{23}^{2} & 0 & r_{34}^{2} & r_{35}^{2} \\
1 & r_{14}^{2} & r_{24}^{2} & r_{34}^{2} & 0 & r_{45}^{2} \\
1 & r_{15}^{2} & r_{25}^{2} & r_{35}^{2} & r_{45}^{2} & 0
\end{array}\right)=0
$$

which we included in our set of equations.

Central configurations satisfy the equation $U / I=-\Lambda M$, where $I$ is the moment of inertia $I=\frac{1}{M} \sum_{i<j} m_{i} m_{j} r_{i j}^{2}, M=\sum m_{i}, U=\sum_{i<j} m_{i} m_{j} r_{i j}^{-1}$ (see for example Moeckel 1990). Since we chose $\Lambda=-1$ we have the equation

$$
e_{I U}=U-M I=0
$$

This is redundant, as it is a consequence of the Albouy-Chenciner equations, but was included (in polynomial form, with cleared denominators) since we thought its simplicity and symmetry might be helpful in the analysis of the tropical prevariety.

In summary, in what follows we will be analyzing the ideal $I_{s}:=\left\langle\mathcal{F}, \mathcal{G}, \mathcal{H}, e_{C M}, e_{I U}\right\rangle$.

\section{The tropical prevariety}

We calculated the tropical prevariety of the set of 47 equations described in the previous section, using Gfan (Jensen 2009). There are 576 rays, with 26 distinct types after considering the symmetry of the equations. In addition, there are 50 distinct cases of two-dimensional cones, 27 distinct cases of three-dimensional cones, 11 distinct cases of four-dimensional cones, and 3 distinct cases of five-dimensional cones. The $f$-vector lists the number of cones of each dimension:

$$
(1,576,1620,1420,450) \text {. }
$$

As described at the end of Sect. 2, we can restrict our attention to those rays and cones which contain vectors $\omega \in \mathbb{R}^{n}$ such that $\sum_{i} \omega_{i} \geq 0$. This leaves us with a total of 44 cases up to symmetry.

Most of these cases can be easily dismissed by computing a Gröbner basis of the initial forms and saturating with respect to the $r_{i j}$ and $m_{i}$ variables. In many cases, if we eliminate the $r_{i j}$ after saturating we obtain a sum over some subset of the masses (e.g. $m_{1}+m_{2}+m_{3}$ ) within the elimination ideal, which means there are no positive mass solutions within that cone. However, for a few rays and two-dimensional cones there were some nonzero solutions for some choice of masses. Gröbner bases were calculated using Singular (Decker et al. 2010) and Sage (Stein et al. 2010). These and other calculations can be found in the supplementary Sage worksheet for this paper (also available at http://www.d.umn.edu/ mhampton/ FiveBodySpatial.sws).

Representatives of all these exceptional cases are given in Table 1 along with the indices of the rays generating the cone and a relative interior point of the cone. All polynomials listed for a given cone must be satisfied. 
Table 1 Representatives of exceptional cases

\begin{tabular}{lll}
\hline Ray indices & Interior point & Exceptional polynomials \\
\hline$[59]$ & $(1,0,0,0,0,0,0,1,1,0)$ & $m_{1} m_{2}-m_{3} m_{4}-m_{3} m_{5}$ \\
{$[72]$} & $(1,0,0,0,0,0,0,1,1,1)$ & $4 m_{1} m_{2}-\left(m_{3}+m_{4}\right)^{2}-\left(m_{3}+m_{5}\right)^{2}+m_{3}^{2}-2 m_{4} m_{5}$ \\
{$[59,72]$} & $(2,0,0,0,0,0,0,2,2,1)$ & $m_{3}-m_{4}-m_{5}, m_{4}^{2}+2 m_{4} m_{5}+m_{5}^{2}-m_{2} m_{1}$ \\
{$[210]$} & $(1,1,0,0,0,1,0,1,0,0)$ & $m_{1}-m_{4}, m_{2}-m_{3}$ \\
{$[453]$} & $(3,3,0,0,0,3,2,3,2,0)$ & $m_{1}-m_{4}, m_{2}-m_{3}$ \\
{$[210,275]$} & $(2,2,1,0,1,2,0,2,0,0)$ & $m_{1}-m_{4}, m_{2}-m_{3}$ \\
{$[193,210]$} & $(2,2,-2,-2,0,2,0,2,0,-2)$ & $m_{1}-m_{4}, m_{2}-m_{3}$ \\
{$[210,453]$} & $(4,4,0,0,0,4,2,4,2,0)$ & $m_{1}-m_{4}, m_{2}-m_{3}$ \\
{$[268,453]$} & $(4,4,0,0,0,4,3,4,3,0)$ & $m_{1}-m_{4}, m_{2}-m_{3}$ \\
{$[270]$} & $(1,1,1,0,0,0,1,0,1,1)$ & See Sect. 5 \\
{$[275]$} & $(1,1,1,0,1,1,0,1,0,0)$ & See Sect. 6 \\
\hline
\end{tabular}

To obtain the above results in the cases with the exceptional polynomials $m_{1}-m_{4}, m_{2}-m_{3}$ we found a decomposition of a certain polynomial that appears in each of the saturated ideals of those cases (sometimes as a factor within a polynomial with no other positive mass solutions):

$$
\begin{aligned}
Q= & m_{1}^{6} m_{2}^{6}+2 m_{1}^{6} m_{2}^{3} m_{3}^{3}+m_{1}^{6} m_{3}^{6}+2 m_{1}^{3} m_{2}^{6} m_{4}^{3}-12 m_{1}^{3} m_{2}^{3} m_{3}^{3} m_{4}^{3} \\
& \quad+2 m_{1}^{3} m_{3}^{6} m_{4}^{3}+m_{2}^{6} m_{4}^{6}+2 m_{2}^{3} m_{3}^{3} m_{4}^{6}+m_{3}^{6} m_{4}^{6} \\
= & \left(m_{1}^{3}-m_{4}^{3}\right)^{2}\left(m_{2}^{3}-m_{3}^{3}\right)^{2}+4 m_{2}^{3} m_{3}^{3}\left(m_{1}^{3}-m_{4}^{3}\right)^{2}+4 m_{1}^{3} m_{4}^{3}\left(m_{2}^{3}-m_{3}^{3}\right)^{2} .
\end{aligned}
$$

This decomposition makes it clear that for real masses $Q=0$ is equivalent to $m_{1}=m_{4}$ and $m_{2}=m_{3}$.

\section{A troublesome ray}

In this section we explain the possible exceptions to the finiteness result stemming from the ray with weight $\omega_{270}=(1,1,1,0,0,0,1,0,1,1)$.

Recall that $I_{s}=\left\langle\mathcal{F}, \mathcal{G}, \mathcal{H}, e_{C M}, e_{I U}\right\rangle$. This is an ideal in $\mathbb{R}\left[r_{12}, \ldots, r_{45}\right]$. Our goal is to show that $J:=\left(\operatorname{in}_{\omega_{270}}\left(I_{S}\right): r_{12} \cdots r_{45}^{\infty}\right)$ contains a monomial, or equivalently, that it is equal to $\langle 1\rangle$.

We first consider the initial forms of the 15 Dziobek equations, $\mathcal{H}$. Six of these are binomials. Among these are $r_{12}^{3} r_{13}^{3} r_{34}^{3}-r_{12}^{3} r_{13}^{3} r_{24}^{3}$ and $r_{13}^{3} r_{14}^{3} r_{24}^{3}-r_{13}^{3} r_{14}^{3} r_{23}^{3}$. We conclude that $r_{34}^{3}-r_{24}^{3}$ and $r_{24}^{3}-r_{23}^{3}$ are in $J$. Similarly, the initial form $-r_{13}^{3} r_{25}^{3}+r_{13}^{3} r_{23}^{3} r_{25}^{3}+r_{13}^{3} r_{15}^{3} r_{25}^{3}$ shows that $r_{23}^{3}+r_{15}^{3}-1$ is in $J$.

We now define $J^{\prime}=\left\langle\right.$ initial forms of $\left.\left(\mathcal{F}, \mathcal{G}, e_{C M}, e_{I U}\right)\right\rangle+\left\langle r_{34}^{3}-r_{24}^{3}, r_{24}^{3}-r_{23}^{3}, r_{23}^{3}+\right.$ $\left.r_{15}^{3}-1\right\rangle \subseteq J$. Performing the substitutions $r_{23}^{3} \mapsto x, r_{24}^{3} \mapsto x, r_{34}^{3} \mapsto x, r_{15}^{3} \mapsto 1-x$ on the generators of $J^{\prime}$ and removing monomial factors, we get a new ideal whose generators do not contain $r_{23}, r_{24}, r_{34}$ and $r_{15}$. Thus instead of considering $J^{\prime}$, we consider $\tilde{J}^{\prime} \subseteq \mathbb{R}\left[r_{13}, r_{14}, r_{25}, r_{35}, r_{45}, x\right]$. This ideal is easy to handle. We compute

$$
\begin{aligned}
& \left(\tilde{J}^{\prime}: r_{12} r_{13} r_{14} r_{25} r_{35} r_{45} x\left(m_{1}+m_{5}\right) m_{5}^{\infty}\right) \cap \mathbb{Q}\left[m_{1}, m_{5}, r_{13}, r_{14}, r_{35}, r_{45}\right] \\
& \quad=\left\langle m_{1} r_{14}^{2}+m_{5} r_{45}^{2}, m_{1} r_{13}^{2}+m_{5} r_{35}^{2}, r_{14}^{2} r_{35}^{2}-r_{13}^{2} r_{45}^{2}\right\rangle,
\end{aligned}
$$


where we regard $\tilde{J}^{\prime}$ as an ideal in $\mathbb{Q}\left[m_{1}, \ldots, m_{5}, r_{13}, r_{14}, r_{25}, r_{35}, r_{45}, x\right]$. For every positive real choice of masses, we conclude that $m_{1} r_{14}^{2}+m_{5} r_{45}^{2}, m_{1} r_{13}^{2}+m_{5} r_{35}^{2}, r_{14}^{2} r_{35}^{2}-$ $r_{13}^{2} r_{45}^{2} \in J$. Similarly, by choosing different subrings for the intersection we get that $\left\{m_{1} r_{12}^{2}+m_{5} r_{25}^{2}, r_{14}^{2} r_{25}^{2}-r_{12}^{2} r_{45}^{2}, r_{13}^{2} r_{25}^{2}-r_{12}^{2} r_{35}^{2}\right\} \subseteq J$.

Now we define $K=\left\langle m_{1} r_{12}^{2}+m_{5} r_{25}^{2}, m_{1} r_{14}^{2}+m_{5} r_{45}^{2}, m_{1} r_{13}^{2}+m_{5} r_{35}^{2}, r_{14}^{2} r_{35}^{2}-r_{13}^{2} r_{45}^{2}, r_{14}^{2} r_{25}^{2}-\right.$ $\left.r_{12}^{2} r_{45}^{2}, r_{13}^{2} r_{25}^{2}-r_{12}^{2} r_{35}^{2}\right\rangle$. Let $d_{1}, d_{2}, d_{3}$ be the initial forms of the Dziobek equations which are not binomial or trinomials - there are only three up to sign. The ideals $\left(\left(\left(\left\langle d_{i}+K\right\rangle\right) \cap\right.\right.$ $\left.\left.\mathbb{Q}\left[m_{1}, m_{5}, r_{12}, r_{13}, r_{14}\right]\right): r_{12} r_{13} r_{14}\left(m_{1}^{3}+m_{5}^{3}\right) m_{1}^{\infty}\right)$ equal

$$
\begin{gathered}
\left\langle\left(r_{12}^{3}-r_{14}^{3}\right)\left(m_{1}\left(r_{12}^{3}-r_{14}^{3}\right)^{2}+m_{5}\left(r_{12}^{3}+r_{14}^{3}\right)^{2}\right)\right\rangle \\
\left\langle\left(r_{13}^{3}-r_{14}^{3}\right)\left(m_{1}\left(r_{13}^{3}-r_{14}^{3}\right)^{2}+m_{5}\left(r_{13}^{3}+r_{14}^{3}\right)^{2}\right)\right\rangle \\
\left\langle\left(r_{12}^{3}-r_{13}^{3}\right)\left(m_{1}\left(r_{12}^{3}-r_{13}^{3}\right)^{2}+m_{5}\left(r_{12}^{3}+r_{13}^{3}\right)^{2}\right)\right\rangle .
\end{gathered}
$$

We conclude that each of these generators, denoted $k_{i}$, are in $J$ for every choice of positive masses. Similarly, $\left(\left(\tilde{J}^{\prime} \cap \mathbb{Q}\left[r_{12}, r_{13}, r_{14}, r_{12}\right]\right):\left(m_{1}+m_{5}\right)^{\infty}\right)=\left\langle m_{2} r_{12}^{2}+m_{3} r_{13}^{2}+m_{4} r_{14}^{2}\right\rangle$ shows that $J$ contains $m_{2} r_{12}^{2}+m_{3} r_{13}^{2}+m_{4} r_{14}^{2}$ for every positive choice of masses.

So it suffices to consider the ideal $\left\langle m_{2} r_{12}^{2}+m_{3} r_{13}^{2}+m_{4} r_{14}^{2}, k_{1}, k_{2}, k_{3}\right\rangle$ homogeneous in $r_{12}, r_{13}$, and $r_{14}$, homogeneous in $m_{1}$ and $m_{5}$, and homogeneous in $m_{2}, m_{3}$, and $m_{4}$. If we dehomogenize by setting $r_{14}=1$ and $m_{5}=1$ we obtain the following:

$$
\begin{gathered}
\left(r_{12}^{3}-1\right)\left(r_{12}^{6}+2 \frac{1-m_{1}^{3}}{1+m_{1}^{3}} r_{12}^{3}+1\right)=0 \\
\left(r_{13}^{3}-1\right)\left(r_{13}^{6}+2 \frac{1-m_{1}^{3}}{1+m_{1}^{3}} r_{13}^{3}+1\right)=0 \\
\left(r_{12}^{3}-r_{13}^{3}\right)\left(r_{12}^{6}+2 \frac{1-m_{1}^{3}}{1+m_{1}^{3}} r_{12}^{3} r_{13}^{3}+r_{13}^{6}\right)=0 \\
r_{12}^{2} m_{2}+r_{13}^{2} m_{3}+m_{4}=0
\end{gathered}
$$

Equations (8) and (9) imply that both $r_{12}$ and $r_{13}$ lie on the unit circle at one of nine points, either at a root of unity or at one of the six locations given by the formula below, in which the choice of cube root is unspecified:

$$
\left(\frac{m_{1}^{3}-1 \pm 2 I \sqrt{m_{1}^{3}}}{m_{1}^{3}+1}\right)^{1 / 3}
$$

These roots are all distinct for positive $m_{1}$.

Equation (10) imposes the further constraint that the ratio of the roots chosen for $r_{12}$ and $r_{13}$ must also be equal to one of the nine points described above; this in turn means that at least one of $r_{12}$ and $r_{13}$ must be a complex root of unity. In addition, the positivity of the masses combined with Eq. (11) means that the imaginary parts of $r_{12}^{2}$ and $r_{13}^{2}$ must have opposite signs.

The consequence of this is that for each choice of $m_{1} / m_{5}$ there are finitely many choices for $m_{2} / m_{4}$ and $m_{3} / m_{4}$. By choosing both $r_{12}$ and $r_{13}$ to be roots of unity with opposing imaginary parts, we always have $m_{2}=m_{3}=m_{4}$ as a solution. In Fig. 1 we show the other possibilities by normalizing $m_{2}+m_{3}+m_{4}=1$ and projecting the set of positive mass solutions orthogonally onto the plane: 
Fig. 1 Exceptional cases for ray 270 in the plane $m_{2}+m_{3}+m_{4}=1$

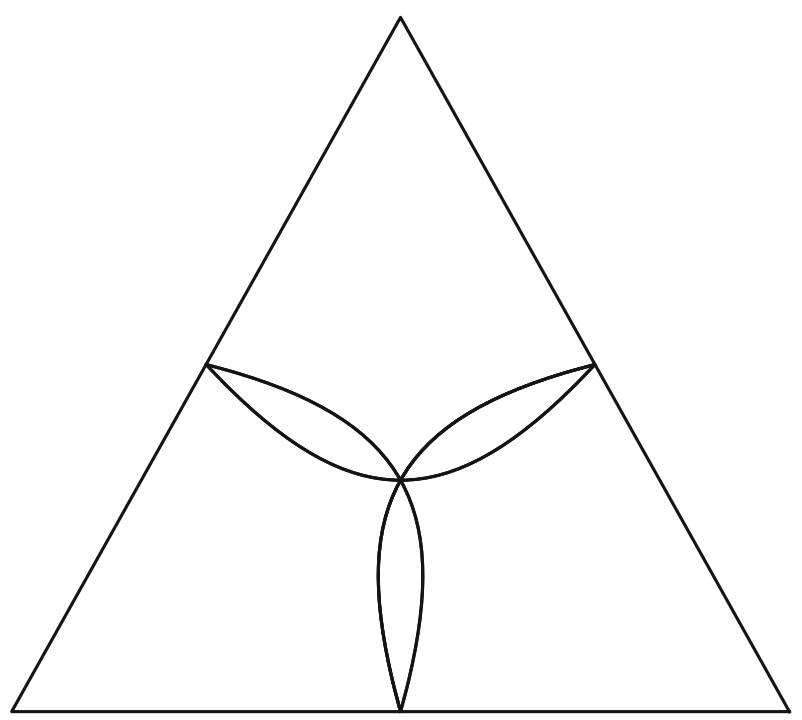

It is straightforward to obtain an explicit polynomial in the masses by eliminating the distance variables, but this polynomial is rather large and unwieldy; we believe the structure of the real solutions is much clearer in the above system.

\section{An even more troublesome ray}

In this section we explain the possible exceptions to the finiteness result stemming from the ray with weight $\omega_{275}=(1,1,1,0,1,1,0,1,0,0)$.

The initial forms of three of the Dziobek equations give us equations of the form

$$
r_{i j}^{-3}+r_{k l}^{-3}-r_{i k}^{-3}-r_{j l}^{-3}=0
$$

for all choices $\{i, j, k, l\}=\{1,2,3,4\}$.

After removing common monomial factors, the remaining equations are binomials of the form $r_{r 5}^{3}-r_{s 5}^{3}$. Using these binomials we may rewrite the Albouy-Chenciner initial form $\operatorname{in}_{\omega_{275}}\left(f_{i 5}\right)$ as $\sum_{j \notin\{i, 5\}} m_{j} r_{i j}^{2}$. This works for $i=1, \ldots, 4$.

Taking these four equations and multiplying the Dziobek equations by monomials, we get the following ideal in $\mathbb{Q}\left[m_{1}, \ldots, m_{4}, r_{13}, \ldots, r_{34}\right]$ :

$$
\begin{aligned}
J^{\prime}:=\langle & m_{4} r_{14}^{2}+m_{3} r_{13}^{2}+m_{2} r_{12}^{2}, m_{4} r_{24}^{2}+m_{3} r_{23}^{2}+m_{1} r_{12}^{2}, \\
& m_{4} r_{34}^{2}+m_{2} r_{23}^{2}+m_{1} r_{13}^{2}, m_{3} r_{34}^{2}+m_{2} r_{24}^{2}+m_{1} r_{14}^{2}, \\
& -r_{13}^{3} r_{24}^{3} r_{34}^{3}+r_{12}^{3} r_{24}^{3} r_{34}^{3}+r_{12}^{3} r_{13}^{3} r_{34}^{3}-r_{12}^{3} r_{13}^{3} r_{24}^{3}, \\
& -r_{14}^{3} r_{23}^{3} r_{24}^{3}+r_{13}^{3} r_{23}^{3} r_{24}^{3}+r_{13}^{3} r_{14}^{3} r_{24}^{3}-r_{13}^{3} r_{14}^{3} r_{23}^{3}, \\
& \left.-r_{14}^{3} r_{23}^{3} r_{34}^{3}+r_{12}^{3} r_{23}^{3} r_{34}^{3}+r_{12}^{3} r_{14}^{3} r_{34}^{3}-r_{12}^{3} r_{14}^{3} r_{23}^{3}\right\rangle
\end{aligned}
$$


The initial form of the Cayley-Menger determinant $e_{C M}$ has four factors:

$$
\begin{aligned}
& \left(-r_{14} r_{23}-r_{13} r_{24}+r_{12} r_{34}\right)\left(-r_{14} r_{23}+r_{13} r_{24}+r_{12} r_{34}\right) \\
& \quad\left(r_{14} r_{23}-r_{13} r_{24}+r_{12} r_{34}\right)\left(r_{14} r_{23}+r_{13} r_{24}+r_{12} r_{34}\right)=0
\end{aligned}
$$

The first three of these factors are equivalent under permutations of $(1,2,3,4)$, so we will only analyze the cases in which the first or last factor vanish.

We consider the ideals $J^{\prime}+\left\langle r_{14} r_{23}+r_{13} r_{24}-r_{12} r_{34}\right\rangle$ and $J^{\prime}+\left\langle r_{14} r_{23}+r_{13} r_{24}+r_{12} r_{34}\right\rangle$. We wish to saturate with respect to all variables and thereafter eliminate the $r$-variables. Since our ideals are homogeneous in the grading of the masses and in the grading of the $r$-variables, any reduced Gröbner basis will work as an elimination Gröbner basis and our difficulty will be to saturate the variables rather than eliminating. The saturation can be carried out in Singular using the technique described in Sturmfels (1996, Lemma 12.1) for computing saturations by reverse lexicographic term orders. Saturating with respect to the variables in the following order worked out for us:

$$
m_{4}, m_{3}, r_{34}, r_{24}, r_{23}, r_{14}, r_{12}, r_{13}, m_{1}, m_{2} \text {. }
$$

In each case after computing the elimination ideal, we are left with a single polynomial in the cubes of the masses. For simplicity we will use $M_{i}=m_{i}^{3}$ as variables. The polynomial is homogeneous of degree 12, with a maximum degree of 6 in each variable. For the case in which $-r_{14} r_{23}-r_{13} r_{24}+r_{12} r_{34}=0$ we will denote this polynomial by $P_{1}$, and in the case $r_{14} r_{23}+r_{13} r_{24}+r_{12} r_{34}$ the polynomial will be denoted by $P_{2}$.

Lemma 1 The only real positive solutions for $P_{1}$ are when

$$
\begin{aligned}
& \left(M_{1}^{2} M_{2}^{2}+M_{1}^{2} M_{4}^{2}+M_{2}^{2} M_{3}^{2}+M_{3}^{2} M_{4}^{2}\right) \\
& \quad-2\left(M_{1} M_{2}^{2} M_{3}+M_{2} M_{3}^{2} M_{4}+M_{1} M_{3} M_{4}^{2}+M_{1}^{2} M_{2} M_{4}\right)-12 M_{1} M_{2} M_{3} M_{4}=0
\end{aligned}
$$

and either $M_{1}=M_{2}$ or $M_{3}=M_{4}$. For real masses this implies that either $m_{1}=m_{2}$ or $m_{3}=m_{4}$.

Proof We will prove the result using interval arithmetic. Because $P_{1}$ is homogeneous and symmetric under the interchanges $M_{1} \leftrightarrow M_{2}, M_{3} \leftrightarrow M_{4}$, and $\left(M_{1}, M_{2}\right) \leftrightarrow\left(M_{3}, M_{4}\right)$, we can dehomogenize by letting $M_{4}=1-M_{1}-M_{2}-M_{3}$ and restrict to the set $\Omega$ given by $0 \leq M_{1} \leq M_{2} \leq 1-M_{1}-M_{2}-M_{3}$ and $M_{3} \leq 1-M_{1}-M_{2}-M_{3}$. It was convenient to decompose this set into three tetrahedra $\Omega_{A}, \Omega_{B}$, and $\Omega_{C}$ with $M_{2} \leq M_{3}, M_{1} \leq M_{3} \leq M_{2}$, and $M_{3}<M_{1}$, respectively.

Our goal is to prove that $P_{1}>0$ in the interior of $\Omega$. Our strategy is to show that the only critical points of $P_{1}$ in the interior of $\Omega$ are positive. $P_{1}$ factors nicely on the boundary of $\Omega$ and there it is easy to see that there are the zero-valued curves of minima described in the statement of the lemma.

To examine the critical points in $\Omega$ with interval arithmetic is somewhat challenging due to the degeneracy of the zero set on its boundary, so we first applied the linear transformation $A$ taking $M$-coordinates to $y$-coordinates according to the identities $M_{1}=y_{1} / 4$, $M_{2}=y_{2} / 3+y_{1} / 4, M_{3}=1 / 2-y_{1} / 4-y_{2} / 6-y_{3} / 2$. The tetrahedron $\Omega_{A}$ maps to the tetrahedron $\hat{\Omega}_{A}$ given by $y_{i} \geq 0, y_{1}+y_{2}+y_{3} \leq 1$. The key advantage of this transformation is that the zero set of $\hat{P}_{1}\left(y_{1}, y_{2}, y_{3}\right)=P_{1}\left(A^{-1}\left(y_{1}, y_{2}, y_{3}\right)\right)$ is contained in the planes $y_{2}=0$ and $y_{3}=0$. This property causes the resultants $R_{12}=\operatorname{Res}_{y_{1}}\left(\partial \hat{P}_{1} / \partial y_{1}, \partial \hat{P}_{1} / \partial y_{2}\right)$ and $R_{23}=\operatorname{Res}_{y_{1}}\left(\partial \hat{P}_{1} / \partial y_{2}, \partial \hat{P}_{1} / \partial y_{3}\right)$ in $\mathbb{C}\left[y_{2}, y_{3}\right]$ to have monomial factors of high degree instead of more complicated polynomial factors. 
Some of the factors of these resultants can be seen to have no zeros in the projection of $\hat{\Omega}$ to the $\left(y_{2}, y_{3}\right)$ plane. For example, $R_{12}$ factorizes as

$y_{2}^{5} y_{3}^{4}\left(y_{2}-3 y_{3}-3\right)^{2}\left(y_{2}-3 y_{3}+3\right)^{2}\left(y_{2}+3 y_{3}-3\right)^{2}\left(y_{2}+3 y_{3}+3\right)^{2}\left(y_{2}^{2}+9 y_{3}^{2}-9\right)^{2} f_{1} f_{2}$

where $f_{1}$ has degree 24 and $f_{2}$ has degree 48 . It is straightforward to show that all the factors other than $f_{1}$ and $f_{2}$ have no zeros in $\hat{\Omega}$.

We use recursive interval arithmetic applied to $\hat{P}_{1}$, the derivatives of $\hat{P}_{1}$, and factors of the above resultants to exclude the existence of non-positive critical values in the interior of $\Omega$ except for very small neighborhoods of the two critical points $\left(M_{1}, M_{2}, M_{3}\right) \in$ $\{(0,0,0),(1 / 3,1 / 3,0)\}$ where $P_{1}$ is zero. For a given polynomial in $n$-variables $x_{1}, \ldots, x_{n}$ we first view it in the ring $\mathbb{Q}\left[x_{1}, \ldots, x_{n-1}\right]\left[x_{n}\right]$ and obtain lower and upper bounds to the coefficients (which are single-variable polynomials in $x_{n}$ ) by verifying numerical estimates with the real root isolation code of Carl Witty in Sage (Witty 2009). The remaining very small neighborhoods can be excluded by considering expansions of the second derivatives of $P_{1}$ around these points.

Lemma 2 The only real positive solutions for $P_{2}$ are when three masses are equal.

Proof Define

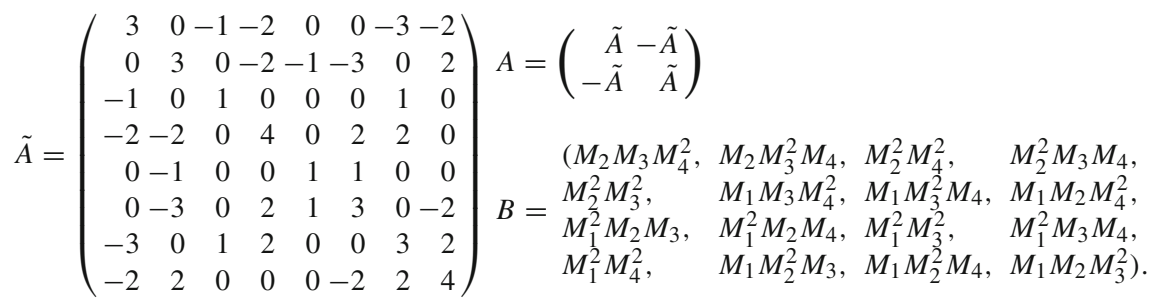

Since $A$ is symmetric with real entries, it is diagonalizable with real eigenvalues by an orthogonal matrix $S$. All eigenvalues are non-negative, since the characteristic polynomial of $A$ is $\lambda^{12}\left(\lambda^{2}-22 \lambda+64\right)^{2}$. Writing the square roots of the eigenvalues in a diagonal matrix $D$ we get $S^{-1} D D S=A$. Consequently, the polynomial $F:=\left(B A B^{T}\right)_{11}$ is the sum of the squares of the polynomial entries of $D S B^{T}$.

The following six polynomials are non-negative on $\mathbb{R}^{4}$ :

$$
\begin{aligned}
D:= & \left(M_{3}-M_{4}\right)^{2}\left(M_{1}-M_{2}\right)^{2}\left(M_{1} M_{2}-M_{3} M_{4}\right)^{2} \\
C:= & F+54 D, \quad E_{1}:=C \cdot\left(M_{3}-M_{4}\right)^{2}\left(M_{1}-M_{2}\right)^{2} \\
E_{2}:= & E_{1}\left(M_{2}, M_{3}, M_{1}, M_{4}\right), \quad E_{3}:=E_{1}\left(M_{1}, M_{3}, M_{2}, M_{4}\right) \\
Y:= & \left(\left(M_{1}-M_{2}\right)^{2}\left(M_{3}-M_{4}\right)^{2}+\left(M_{1}-M_{3}\right)^{2}\left(M_{2}-M_{4}\right)^{2}\right. \\
& \left.+\left(M_{1}-M_{4}\right)^{2}\left(M_{3}-M_{2}\right)^{2}\right)^{2}
\end{aligned}
$$

The following polynomial is positive on $\mathbb{R}_{>0}^{4}$ unless $M_{1}=M_{2}=M_{3}=M_{4}$ :

$$
\begin{aligned}
X= & M_{1} M_{2}\left(M_{3}-M_{4}\right)^{2}+M_{1} M_{3}\left(M_{2}-M_{4}\right)^{2}+M_{1} M_{4}\left(M_{2}-M_{3}\right)^{2} \\
& +M_{2} M_{3}\left(M_{1}-M_{4}\right)^{2}+M_{2} M_{4}\left(M_{1}-M_{3}\right)^{2}+M_{3} M_{4}\left(M_{1}-M_{2}\right)^{2}
\end{aligned}
$$

Our polynomial $P_{2}$ equals $\frac{1}{2}\left(E_{1}+E_{2}+E_{3}+4 X Y\right)$. Hence, if $P_{2}\left(M_{1}, M_{2}, M_{3}, M_{4}\right)$ is 0 with $M_{1}, M_{2}, M_{3}$ and $M_{4}$ in the positive orthant, then all masses must be equal, or $Y\left(M_{1}, M_{2}, M_{3}, M_{4}\right)$ must be 0 . This happens only when three masses are equal. 
For the shape of the decomposition of $P_{2}$ in the proof above we were inspired by the Real Nullstellensatz (Stengle 1974, see also Sturmfels 2002, Theorem 7.5). One region on which we want to show that $P_{2}$ is positive in the proof can be described by six inequalities: $M_{i}>0,\left(M_{1}-M_{2}\right)^{2}>0$, and $\left(M_{3}-M_{4}\right)^{2}>0$. The Real Nullstellensatz essentially says that if our lemma is true then a multiple of $P_{2}$ can be written as a linear combination of the $2^{6}$ products of inequality constraints, with the coefficients being polynomials which are sums of squares. The theorem gives no reasonable degree bound on the coefficients, but for fixed Newton polytopes of the coefficients, the existence of the expression can be decided by SOSTOOLS (Prajna et al. 2004) using semidefinite programming. The answer from SOSTOOLS is unreliable as floating point computations are used, but SOSTOOLS is an extremely useful tool for suggesting which inequality products are needed, and validating simplified alternative shapes for the decomposition. It is important to enforce symmetries on the decomposition to increase the chances of rounding off floating point numbers to integers consistently during the simplification process. Only when the expression has been sufficiently simplified, SOSTOOLS will give an integer certificate (like $A$ above). Due to the smaller group of symmetries of $P_{1}$ we were unable to find a similar integer decomposition of this polynomial but propose it as an exciting challenge.

\section{Conclusion}

We have shown that the Newtonian five-body problem has finitely many spatial central configurations, apart from some explicitly given possible exceptional cases for certain values of the mass parameters.

It seems likely that the spatial five-body central configurations are always finite for positive masses. Our possible exceptional cases often involve some equality between masses, but there is strong numerical evidence that there are finitely many central configurations in the case of five equal masses (Lee and Santoprete 2009). Presumably the failure of our methods to resolve our exceptional cases merely reflects the existence of additional syzygies in the leading terms of the generators of our ideal.

Acknowledgments Some of our computations were run on one of the Sage Foundation's 24-core Sun X4450s, supported by National Science Foundation Grant No. DMS-0821725. We were also both supported by the American Institute of Mathematics. Furthermore, the second author was supported by the German Research Foundation (Deutsche Forschungsgemeinschaft (DFG)) through the Institutional Strategy of the University of Göttingen.

Open Access This article is distributed under the terms of the Creative Commons Attribution Noncommercial License which permits any noncommercial use, distribution, and reproduction in any medium, provided the original author(s) and source are credited.

\section{References}

Albouy, A., Chenciner, A.: Le problème des n corps et les distances mutuelles. Inv. Math. 131, 151-184 (1998)

Albouy, A., Kaloshin, V.: Personal communication (2010)

Bernstein, D.N.: The number of roots of a system of equations. Funct. Anal. Appl. 9, 183-185 (1975)

Bieri, R., Groves, J.R.J.: The geometry of the set of characters induced by valuations. J. Reine Angew. Math. 347, 168-195 (1984)

Decker, W., Greuel, G.M., Pfister, G., Schönemann, H.: Singular 3-1-1-A computer algebra system for polynomial computations. http://www.singular.uni-kl.de (2010)

Dziobek, O.: Über einen merkwürdigen fall des vielkörperproblems. Astron. Nach. 152, 152 (1900) 
Euler, L.: De motu rectilineo trium corporum se mutuo attrahentium. Novi. Comm. Acad. Sci. Imp. Petrop. 11, 144-151 (1767)

Hampton, M., Moeckel, R.: Finiteness of relative equilibria of the four-body problem. Inv. Math. 163, 289-312 (2006)

Hept, K., Theobald, T.: Tropical bases by regular projections. Proc. Am. Math. Soc. 137, 2233-2241 (2009)

Jensen, A.N.: Algorithmic Aspects of Gröbner Fans and Tropical Varieties. PhD thesis, Department of Mathematical Sciences, University of Aarhus, Denmark (2007)

Jensen, A.N.: Gfan, a software system for Gröbner fans. http://www.math.tu-berlin.de/ jensen/software/gfan/ gfan.html (2009)

Khovanskii, A.G.: Newton polyhedra and toric varieties. Funct. Anal. Appl. 11, 289-296 (1977)

Kushnirenko, A.G.: Newton polytopes and the bézout theorem. Funct. Anal. Appl. 10, 233-235 (1976)

Lagrange, J.L.: Essai sur le problème des trois corps. Oeuvres 6 (1772)

Lee, T.L., Santoprete, M.: Central configurations of the five-body problem with equal masses. Celest. Mech. Dyn. Astron. 104, 369-381 (2009)

Moeckel, R.: On central configurations. Math. Zeit. 205, 499-517 (1990)

Moeckel, R.: Generic finiteness for Dziobek configurations. Trans. Am. Math. Soc. 353, 4673-4686 (2001)

Moulton, F.R.: The straight line solutions of the problem of $n$ bodies. Ann. Math. 12, 1-17 (1910)

Newton, I.: Philosophi Naturalis Principia Mathematica. Royal Society, London, UK (1687)

Prajna, S., Papachristodoulou, A., Seiler, P., Parrilo, P.A.: SOSTOOLS: Sum of squares optimization toolbox for MATLAB (2004)

Roberts, G.: A continuum of relative equilibria in the five-body problem. Phys. D 127, 141-145 (1999)

Roberts, G.: Personal communication (2009)

Speyer, D., Sturmfels, B.: The tropical grassmannian. Adv. Geom. 4, 389-411 (2004)

Stein, W., et al.: Sage Mathematics Software (Version 4.5.2). The Sage Development Team http://www. sagemath.org (2010)

Stengle, G.: A nullstellensatz and a positivstellensatz in semialgebraic geometry. Math. Ann. 207, 87-97 (1974)

Sturmfels, B.: Gröbner Bases and Convex Polytopes. American Mathematical Society, Providence, R.I. (1996)

Sturmfels, B.: Solving systems of polynomial equations, CBMS Regional Conference Series in Mathematics, vol 97. Published for the Conference Board of the Mathematical Sciences, Washington, DC (2002)

Witty, C.: Isolate Real Roots of Real Polynomials, http://www.sagemath.org/doc/reference/sage/rings/ polynomial/real_roots.html (2009)

Zeigler, G.: Lectures on Polytopes, Graduate Texts in Mathematics, vol. 152. Springer, New York (1995) 